\title{
IMPACT OF MOISTURE STRESS AND NITROGEN ON CROP GROWTH RATE, NITROGEN USE EFFICIENCY, AND HARVEST INDEX OF COTTON (Gossypium hirsutum L.)
}

\author{
Mahmood-ul-Hassan", ${ }^{1,}$ Muhammad Maqsood², Syed Aftab Wajid ${ }^{2}$ and Atta Muhammad \\ Ranjha $^{3}$
}

\author{
${ }^{1}$ Nuclear Institute for Agriculture and Biology (NIAB), Faisalabad, Pakistan; ${ }^{2}$ Department of Agronomy, University \\ of Agriculture, Faisalabad, Pakistan; ${ }^{3}$ Institute of Soil and Environmental Sciences, University of Agriculture, \\ Faisalabad, Pakistan. \\ *Corresponding author's e-mail : mhassan@niab.org.pk, mhj407jb@yahoo.com
}

\begin{abstract}
Nitrogen and moisture stress generally modify growth and development of cotton like several other field crops. Field experiments were conducted for two years to elucidate the interactive effect of moisture stress (I), nitrogen (N), and genotype (V) on agronomic traits, nitrogen use efficiency (NUE) and harvest index (HI) of cotton by using RCBD split-split plot experimental design. Four moisture stress treatments $\left(\mathrm{I}_{1}\right.$ : no stress, $\mathrm{I}_{2}$ : moisture stress at inter-node elongation stage, $\mathrm{I}_{3}$ : moisture stress at vegetative growth stage, and $\mathrm{I}_{4}$ : moisture stress at inter-node elongation and vegetative growth stages), three nitrogen levels $\left(50-\mathrm{N}_{1}, 100-\mathrm{N}_{2}\right.$, and $150 \mathrm{~kg} \mathrm{ha}^{-1}-\mathrm{N}_{3}$ ), and three genotypes of cotton (NIAB-846- $\mathrm{V}_{1}$, NIAB-824- $\mathrm{V}_{2}$, and CIM-496) were used in this study. Moisture stress, nitrogen, and cotton genotypic interaction exhibited significant effect on number of bolls per plant, leaf area duration (LAI), crop growth rate (CGR), net assimilation rate (NAI), NUE, and HI. In all genotypes of cotton, highest CGR, NAI, NUE, and HI was recorded where irrigation was applied at all growth stages (in no stress treatment) and moisture stress at vegetative growth stage (in treatment where irrigation was withheld at vegetative growth stage) with $\mathrm{N}$ dose of $50 \mathrm{~kg} \mathrm{ha}^{-1}$ expect in few treatments where $150 \mathrm{~kg} \mathrm{~N} \mathrm{ha}^{-1}$ exhibited greater crop growth rate. During $1^{\text {st }}$ year field experiment highest increase in HI of; NIAB- 846 was $10.46 \%$ by $\mathrm{I}_{3} \times \mathrm{N}_{1} \times \mathrm{V}_{1}$, NIAB- 824 was $80.62 \%$ by $\mathrm{I}_{3} \times \mathrm{N}_{3} \times \mathrm{V}_{2}$, and CIM496 was $33.91 \%$ by $\mathrm{I}_{3} \times \mathrm{N}_{3} \times \mathrm{V}_{3}$. During $2^{\text {nd }}$ year, highest increase in HI was $31.60 \%$ in NIAB-846 (by $\mathrm{I}_{3} \times \mathrm{N}_{3} \times \mathrm{V}_{1}$ ), $20.98 \%$ in NIAB-824 (by $I_{1} \times N_{1} \times V_{2}$ ), and 20.10\% in CIM-496 (by $I_{1} \times N_{2} \times V_{3}$ ). Nevertheless, highest NUE was achieved in no stress treatment but moisture stress at vegetative growth stage didn't significantly reduce harvest index and nitrogen use efficiency as compared to no stress (well water) treatment. Thus it may be concluded that moisture stress at vegetative growth stage in these cotton genotypes could be applied for saving of irrigation water and nitrogen fertilizer.
\end{abstract}

Keywords: Cotton, crop growth rate, harvest index, moisture stress, nitrogen use efficiency.

\section{INTRODUCTION}

Nitrogenous fertilizers and irrigation water are essential inputs for sustainable production of cotton. Water scarcity due to global environmental changes, sharply increasing costs of fertilizers, and intensive cropping demands for judicious use of these inputs by use of improved management practices. Field crops' yield including cotton has been adversely affected by shortage of irrigation water in recent years (Dagdelen et al., 2009; Singh et al., 2010). Wastage of irrigation water and mismanagement in nitrogenous fertilizers application in cotton has also increased its cost of production (Devkota et al., 2013).

In Pakistan, importance of water saving technologies has been recognized by many scientist for judicious use of limited river water supplies (Buttar et al., 2007) that are further depleting day by day. It is great challenge for the scientists to generate new knowledge for better understanding of changes in agronomic and physiological traits of cotton under multiple stresses especially moisture and nutrients stress in the field. Environmental variations and drought has caused greater changes in genotype $\mathrm{x}$ year and/or genotype $\mathrm{x}$ location interactions of cotton (Islam et al., 2013; Gul et al., 2014). Even under favorable environment with higher nitrogen, the yield progress was not up to the mark (Howard et al., 2001). A shift in production technologies is required for sustainable yield improvement under both the conditions. Injudicious use of irrigation water and nitrogenous fertilizers are major causes of lower average yield of seed cotton in Pakistan. Depletion of irrigation resources and ever increasing costs of fertilizers have further enhanced the importance of improvement in management practices for saving of irrigation water and fertilizers. The problem of irrigation water shortage has become a global (Clay et al., 2001). In spite of the importance of water shortage, the research output of water saving technologies is negligible. There are several reasons of this shortcoming. Firstly, the traditional diagnostic tools that are being used for measurement of water use (e.g. plant 
transpiration and stomatal conductance) are point measurements. Secondly, the experimental approaches for direct measurement of water (i.e., weighing lysimeters) are expensive to build, operate, and maintain. Thirdly, it is general assumption by several scientists that management recommendations should not depend on unpredictable climatic conditions. So it is very important to develop water saving technologies. Over-irrigation and insufficient irrigation both may be drastic for crops (Fereres et al., 2007). Over-irrigation in cotton causes more vegetative growth, nutrients leaching, and contamination of ground water. On the other hand insufficient irrigation may affect plant growth, less fruiting and lower yield. It is a matter of great concern to increase the nutrients and water use efficiency of cotton under diminishing water resources. Although several components of soil-plant system can be managed but the effects on water use efficiency is not consistent across locations and experiments (Hatfield et al., 2001). The main reason may be the lack of information addressing the response of cotton plants to varietal-specific multiple stresses in the field. Due to important cash crop, several scientists have made efforts to increase water use efficiency of cotton (Tennakoon and Milroy, 2003; Tang et al., 2005); however, the knowledge about genotypic-specific response of cotton on alkaline calcareous soils under moisture stress and nitrogen interaction effects is rare. In Pakistan cotton is grown on $>3$ million hectares under extreme climatic conditions of high temperature ranging $28-50^{\circ} \mathrm{C}$ on soils which are alkaline calcareous in nature with problems of high nutrients losses coupled with brackish under-ground water that is not fit for irrigation. In this view it was imperative to investigate the changes in plant growth, nitrogen use efficiency, and harvest index of cotton by moisture stress, nitrogen, and genotypic interaction; to test the hypothesis that whether we can save irrigation water and nitrogen by imposing moisture stress at certain growth stages without any significant yield loss.

\section{MATERIALS AND METHODS}

Experimental sites and treatments detail: Field experiments were conducted for two consecutive years (2008 to 2010) at Nuclear Institute for Agriculture and Biology (NIAB), Faisalabad, Pakistan situated in mixed cropping zone of country. Soil properties were examined prior to field experiment each year. Experimental fields were medium loam with greater proportion of sand and silt, alkaline calcareous in nature with low organic matter. Meteorological data of the experimental fields were also recorded at the nearest observatory (situated about $500 \mathrm{~m}$ away from experimental plots). Randomized complete block design with split-split plot arrangements in triplicate was used in these experiments. Plot size was kept $3.6 \times 3.0 \mathrm{~m}$. Three factors; moisture stress, nitrogen, and genotype were used in this study. Moisture stress treatments used were: no stress = eight irrigations as recommended by Government Agricultural Extension Departments, all irrigations were applied at $50 \%$ available soil moisture depletion level (ASMDL)- $\mathrm{I}_{1}$; moisture stress at inter-node elongation stage $=$ withholding irrigation in July up to $80 \%$ ASMDL-I $\mathrm{A}_{2}$; moisture stress at vegetative growth stage $=$ withholding irrigation in September up to $80 \%$ ASMDL- $\mathrm{I}_{3}$; moisture stress at inter-node elongation and vegetative growth stage $=$ withholding irrigation in July and September at $80 \%$ ASMDL-I 4 . Nitrogen treatments were: 50$\mathrm{N}_{1}, 100-\mathrm{N}_{2}$, and $150 \mathrm{~kg} \mathrm{ha}{ }^{-1}-\mathrm{N}_{3}$ (recommended dose for farmers). Urea $(46 \% \mathrm{~N})$ was used as a source of nitrogen in all the treatments. Cotton genotypes grown in these field experiments were; NIAB-846 (V1), NIAB-824 (V2), and CIM-496 (V3).

Table 1. The meteorological data during cotton growing seasons.

\begin{tabular}{|c|c|c|c|c|c|c|c|c|c|}
\hline \multicolumn{2}{|c|}{ Climatic factor/growing season } & May & Jun. & Jul. & Aug. & Sep. & Oct. & Nov. & Dec. \\
\hline \multicolumn{10}{|c|}{ Mean Max. Temperature } \\
\hline \multirow[t]{2}{*}{ Mean Max. Temp. } & 2008-09 & 38.40 & 37.80 & 37.70 & 35.00 & 35.30 & 33.70 & 28.30 & 22.90 \\
\hline & $2009-10$ & 40.10 & 40.90 & 37.90 & 36.50 & 36.10 & 33.40 & 26.30 & 22.90 \\
\hline \multirow[t]{2}{*}{ Highest Max. Temp. } & 2008-09 & 44.50 & 41.60 & 41.00 & 39.40 & 38.30 & 37.30 & 33.60 & 27.40 \\
\hline & $2009-10$ & 44.90 & 45.00 & 43.60 & 40.60 & 38.60 & 37.50 & 32.50 & 27.00 \\
\hline \multirow[t]{2}{*}{ Lowest Temp. } & 2008-09 & 30.50 & 30.60 & 32.20 & 27.70 & 29.00 & 28.00 & 23.60 & 24.00 \\
\hline & $2009-10$ & 31.80 & 34.20 & 32.00 & 26.30 & 25.70 & 28.50 & 23.50 & 19.60 \\
\hline \multicolumn{10}{|c|}{ Mean Min. Temperature } \\
\hline \multirow{2}{*}{ Mean Min. Temp. } & 2008-09 & 23.40 & 26.90 & 27.70 & 26.00 & 23.20 & 19.90 & 10.80 & 7.80 \\
\hline & $2009-10$ & 24.30 & 25.60 & 26.50 & 26.40 & 23.40 & 16.20 & 10.00 & 4.60 \\
\hline \multirow[t]{2}{*}{ Highest Min. Temp. } & 2008-09 & 26.70 & 30.00 & 30.40 & 28.40 & 26.20 & 25.50 & 16.00 & 15.00 \\
\hline & $2009-10$ & 29.50 & 29.50 & 30.00 & 29.70 & 26.30 & 25.10 & 16.80 & 7.80 \\
\hline \multirow[t]{2}{*}{ Lowest Min. Temp. } & 2008-09 & 19.00 & 21.50 & 23.00 & 21.00 & 17.00 & 15.80 & 6.00 & 5.00 \\
\hline & $2009-10$ & 18.90 & 21.80 & 21.40 & 20.00 & 21.00 & 11.00 & 4.00 & 2.30 \\
\hline \multirow[t]{2}{*}{ Rainfall Total } & 2008-09 & 53.90 & 118.20 & 63.40 & 273.00 & 37.00 & - & - & 14.50 \\
\hline & 2009-10 & 10.00 & 5.80 & 52.50 & 137.40 & 30.20 & 14.80 & 0.40 & 0.00 \\
\hline
\end{tabular}


From every plot, one half area was used for recording of growth parameters and destructive use, the remaining was allocated for the final harvest data. Soil water content in root zone was measured by using the gravimetric procedure of direct soil water measurement. The moisture content in root zone was computed by using the method as described by (Penman, 1970; French and Legg, 1979). Soil sampling from experimental plots $(0-60 \mathrm{~cm}$ depth) was done on regular basis on alternate days with the help of soil sampling probe throughout cropping period (May-December) for determination of soil moisture. Soil sampling was done from inter-row spacing to avoid excavation of whole field. Effective root zone depth for moisture extraction in the soil was taken as $150 \mathrm{~cm}$ with exception of $15 \mathrm{~cm}$ surface layer. Oven drying of collected soil samples was made till constant weight at $100^{\circ} \mathrm{C}$. When moisture content in the root zone was reached at desired level, irrigation water was applied to the treatments in measured quantity up to the field capacity. Rainfall received by the experimental plots was recorded and the remaining amount of water in a measured quantity was applied to the treatments at the achieving of desired moisture contents in the root zone as described for different moisture stress treatments. The total amounts of water (irrigation + rainfall) received by different treatments during 2008-09 growing seasons was $873.40 \mathrm{~mm}$ in $\mathrm{I}_{1}, 773.40 \mathrm{~mm}$ in $\mathrm{I}_{2}$, $773.40 \mathrm{~mm}$ in $\mathrm{I}_{3}$, and $673.40 \mathrm{~mm}$ in $\mathrm{I}_{4}$. The same amount of water was applied in these treatments during 2009-10 growing season.

Procedure for recording observations: Agronomic traits and physiological data were recorded from ten randomly selected guarded plants from every treatment. Portable laser leaf area meter (model CI-202, CID Bio-Science Inc.) was used in the field for measurement of leaf area. Leaf area of selected plants was measured, without detaching the leaves from plants and then averaged to get leaf area per plant.

The leaf area index was calculated by using the equation as given below:

$$
\text { Leaf area index }=\frac{\text { Leaf area per plant }}{\text { Land area per plant }}
$$

Leaf area duration is the relationship of leaf area index recorded at different time during growth period. Leaf area duration (LAD) was estimated according to Hunt (1978), as given:

$$
\mathrm{LAD}=\frac{\mathrm{LAI}_{1}+\mathrm{LAI}_{2}}{2} \times \mathrm{t}_{2}-\mathrm{t}_{1}
$$

$\mathrm{LAI}_{1}$ and $\mathrm{LAI}_{2}$ are the leaf area indices at time $\mathrm{t}_{2}$ (October) and $t_{1}$ (September) respectively.

A sample of three plants from every plot was harvested at ground level at 120 days $\left(\mathrm{t}_{1}\right)$ crop (peak fruiting stage) and at 150 days $\left(\mathrm{t}_{2}\right)$ crop (boll maturity stage). The plants components (leaves, fruits, stem, and branches) were separated and oven dried at $70^{\circ} \mathrm{C}$ in air forced oven till constant weight. Total dry matter per plant was obtained by adding weight of all components and average dry weight was calculated. Crop growth rate $\left(\mathrm{g} \mathrm{m}^{-2} \mathrm{day}^{-1}\right)$ was calculated as proposed by Hunt (1978):

$$
\mathrm{CGR}=\frac{\mathrm{W}_{2}-\mathrm{W}_{1}}{\mathrm{t}_{2}-\mathrm{t}_{1}}
$$

Here $\mathrm{W}_{1}$ and $\mathrm{W}_{2}$ are the final dry matter at times $\mathrm{t}_{1}$ and $\mathrm{t}_{2}$, respectively.

Net assimilation rate $\left(\mathrm{g} \mathrm{m}^{-2}\right.$ day $\left.^{-1}\right)$ was calculated as proposed by Hunt (1978):

$$
\mathrm{NAR}=\frac{\mathrm{TDM}}{\mathrm{LAD}}
$$

Here TDM and LAD are the total dry matter per plant and leaf area duration per plant, respectively.

For determination of seed cotton yield plot $^{-1}$, bolls from individual experimental plots were picked manually at $8-10 \%$ moisture contents in seed cotton and then weighed by using electronic balance, Then seed cotton yield was converted to yield $\mathrm{ha}^{-1}$ Total number of bolls on ten selected plants were counted and then averaged to calculate number of bolls per plant. Then plants were harvested at ground level and sundried for one week till $10 \pm 2 \%$ moisture and weighed and averaged to calculate Shoot dry weight per plant Harvest index was calculated by using the following formula:

$$
\text { H.I. }(\%)=\frac{\text { Seed cotton yield } \mathrm{kg} \text { per plant }}{\text { Total shoot dry weight kg per plant }} \times 100
$$

Nitrogen use efficiency (NUE) was calculated as:

$$
\mathrm{NEU}=\frac{\text { Seed cotton yield kg per hectare }}{\text { Nitrogen applied kg per hectare }}
$$

(Thind et al., 2008)

The NUE (kg seed cotton per $\mathrm{kg} \mathrm{N}$ applied) of different treatments was determined by above mentioned method.

Meteorological data: Meteorological data recorded during the crop growing period (Table 1) presents mean monthly values of the temperature and rainfall. Mean values of maximum and minimum temperature during 2008-09 growing season were recorded as $38.40^{\circ} \mathrm{C}$ and $22.90^{\circ} \mathrm{C}$ in the months of May and December, respectively. Whereas mean values of maximum and minimum temperature were found in the months of June and December as $40.90^{\circ} \mathrm{C}$ and $22.90^{\circ} \mathrm{C}$, respectively. Highest maximum temperature ranges were $44.50^{\circ} \mathrm{C}$ during May $2008,45^{\circ} \mathrm{C}$ in June 2009 ; and lowest, temperature ranges were $24^{\circ} \mathrm{C}$ and $19.6^{\circ} \mathrm{C}$ during 2008 and 2009 , respectively. Total rainfall during growing period was $560 \mathrm{~mm}$ during 2008 and $251 \mathrm{~mm}$ during 2009. Both the year maximum rainfall of $273 \mathrm{~mm}$ and $137.4 \mathrm{~mm}$ was recorded in 
the month of August. Total rainfall during last four months of growth period from September to December was $51.5 \mathrm{~mm}$ in 2008 and $45.10 \mathrm{~mm}$ in 2009.

Statistical analysis: Data collected were tabulated and analyzed by Randomized Complete Block 3-factors split-split plot design ANOVA process by using Fisher's analysis of variance technique. Factor effects were considered significant at the $\mathrm{P} \leq 0.05$. Where $\mathrm{F}$ values were significant, means were separated by least significant difference (LSD) at $5 \%$ probability level (Steel and Torrie, 1984). Computer software packages Minitab-15 and Statistix version 8.1 were used for data analysis whereas. Microsoft excel version 2010 was used for preparation of graphs.

\section{RESULTS}

Number of bolls per plant at maturity: The number of bolls per plant varied widely under different $\mathrm{N}$ levels (Table 2) during cropping season 2008-09 but remained non-significant during 2009-10 growing season. Treatments $(\mathrm{I} \times \mathrm{N} \times \mathrm{V})$ exhibited significant variation in number of bolls per plant during two years study. Highest number of bolls per plant (during 2008-09) was observed in treatment $\mathrm{I}_{1} \times \mathrm{N}_{2} \times \mathrm{V}_{1}$ (no stress $\left.\times 100 \mathrm{~kg} \mathrm{~N} \mathrm{ha}^{-1} \times \mathrm{NIAB}-846\right)$ with 47.67 bolls per plant, followed by $\mathrm{I}_{3} \times \mathrm{N}_{3} \times \mathrm{V}_{1}$ (moisture stress at vegetative growth stage $\times 150 \mathrm{~kg} \mathrm{ha}^{-1} \mathrm{~N} \times \mathrm{NIAB}-846$ ) with 44.27 bolls per plant
(Fig. 1a). Similar trend was observed during 2009-10 (Fig. 1b) where maximum number of bolls per plant; 43.17 and 39.73 were recorded in treatments $\mathrm{I}_{1} \times \mathrm{N}_{2} \times \mathrm{V}_{2}$ (no stress $\times 100$ $\mathrm{kg} \mathrm{ha}^{-1} \mathrm{~N} \times \mathrm{NIAB}-824$ ) and $\mathrm{I}_{3} \times \mathrm{N}_{1} \times \mathrm{V}_{1}$ (moisture stress at vegetative growth stage $\times 50 \mathrm{~kg} \mathrm{~N} \mathrm{ha}^{-1} \times$ NIAB-846), respectively.

Leaf area duration: Leaf area duration is the relationship of leaf area index recorded at different time during growth period. Nitrogen and moisture stress markedly affected leaf area duration (LAD). Highest LAD in cotton genotypes was attained by $\mathrm{I}_{1}$ (no stress) and $\mathrm{N}_{3}\left(150 \mathrm{~kg} \mathrm{~N} \mathrm{ha}^{-1}\right)$ during both the years. Among genotypes, highest LAD during 2008-09 was observed in NIAB-824 whereas during 2009-10, NIAB846 showed greater LAD. Interaction $(\mathrm{I} \times \mathrm{N} \times \mathrm{V})$ significantly affected LAD as shown in Fig. 1c,d. During 2008-09 (Fig. 1c), highest LAD (216 d) was recorded by $\mathrm{I}_{1} \times \mathrm{N}_{3} \times \mathrm{V}_{2}$ (no stress $\left.\times 150 \mathrm{~kg} \mathrm{~N} \mathrm{ha}^{-1} \times \mathrm{NIAB}-824\right)$ followed by $213 \mathrm{~d} \mathrm{LAD}$ by $\mathrm{I}_{1} \times \mathrm{N}_{3} \times \mathrm{V}_{1}$ (no stress $\left.\times 150 \mathrm{~kg} \mathrm{~N} \mathrm{ha}^{-1} \times \mathrm{NIAB}-846\right)$ and 210 days LAD by $\mathrm{I}_{1} \times \mathrm{N}_{3} \times \mathrm{V}_{3}$ (no stress $\times 150 \mathrm{~kg} \mathrm{~N} \mathrm{ha}^{-1} \times \mathrm{CIM}-$ 496). During 2009-10 (Fig. 1d), highest LAD of 235 and 234 $\mathrm{d}$ remained in $\mathrm{I}_{1} \times \mathrm{N}_{3} \times \mathrm{V}_{1}$ (no stress $\times 150 \mathrm{~kg} \mathrm{~N} \mathrm{ha}^{-1} \times$ NIAB846) and $I_{2} \times N_{1} \times V_{3}$ (stress at internodes elongation stage $\times$ $50 \mathrm{~kg} \mathrm{~N} \mathrm{ha}^{-1} \mathrm{x}$ CIM-496), respectively. Lowest LAD was found in treatments where moisture stress at two stages (i.e. internodes elongation and at vegetative growth stages) was imposed (Fig. 1 c,d). Lowest LAD of 87 and $94 \mathrm{~d}$ was noted

Table 2. Moisture stress, nitrogen, and genotypes interaction effect on number of bolls per plant, leaf area duration, crop growth rate, net assimilation rate, nitrogen use efficiency, and harvest index of cotton.

\begin{tabular}{|c|c|c|c|c|c|c|c|c|c|c|c|c|}
\hline \multirow[t]{2}{*}{ Factors } & \multicolumn{2}{|c|}{ Bolls per plant } & \multicolumn{2}{|c|}{ LAD (d) } & \multicolumn{2}{|c|}{$\begin{array}{c}\text { CGR } \\
\left(\mathrm{g} \mathrm{m}^{-2} \mathbf{d}^{-1}\right)\end{array}$} & \multicolumn{2}{|c|}{$\begin{array}{c}\text { NAR } \\
\left(\mathrm{g} \mathrm{m}^{-2} \mathbf{d}^{-1}\right)\end{array}$} & \multicolumn{2}{|c|}{$\begin{array}{c}\text { NUE (kg SCY } \\
\text { kg-1 } \mathbf{N} \text { applied) }\end{array}$} & \multicolumn{2}{|c|}{ HI (\%) } \\
\hline & 2008-09 & $2009-10$ & 2008-09 & $2009-10$ & 2008-09 & $2009-10$ & 2008-09 & 2009-10 & $2008-10$ & $2009-10$ & 2008-09 & $2009-10$ \\
\hline \multicolumn{13}{|c|}{ Moisture stress (I) } \\
\hline $\mathrm{I}_{1}$ & $37.70 \mathrm{a}$ & $35.58 \mathrm{a}$ & $184 a$ & $167 \mathrm{a}$ & $5.26 a$ & $4.81 b$ & $3.06 \mathrm{a}$ & $3.16 \mathrm{~b}$ & $51.56 \mathrm{a}$ & $53.01 \mathrm{a}$ & $18.33 b$ & $19.14 \mathrm{a}$ \\
\hline $\mathrm{I}_{2}$ & $33.67 b$ & $32.28 \mathrm{~b}$ & $158 \mathrm{c}$ & $164 \mathrm{a}$ & $1.78 \mathrm{c}$ & $1.49 \mathrm{c}$ & $2.97 \mathrm{~b}$ & $3.28 \mathrm{~b}$ & $36.46 \mathrm{c}$ & $48.37 b$ & $17.64 \mathrm{~b}$ & $17.98 \mathrm{~b}$ \\
\hline $\mathrm{I}_{3}$ & $33.78 b$ & $35.96 \mathrm{a}$ & $168 b$ & $155 b$ & $4.20 \mathrm{~b}$ & $5.63 \mathrm{a}$ & $2.70 \mathrm{~b}$ & $3.61 \mathrm{a}$ & $47.93 b$ & $52.30 \mathrm{a}$ & $23.32 \mathrm{a}$ & $19.68 \mathrm{a}$ \\
\hline $\mathrm{I}_{4}$ & $32.04 \mathrm{~b}$ & $29.47 \mathrm{c}$ & $111 d$ & $143 c$ & $1.51 \mathrm{~d}$ & $1.30 \mathrm{c}$ & $4.11 \mathrm{a}$ & $3.75 \mathrm{a}$ & $32.78 \mathrm{~d}$ & $44.21 \mathrm{c}$ & $14.36 \mathrm{c}$ & $17.90 \mathrm{~b}$ \\
\hline LSD $\mathrm{P}<0.05)$ & $2.68 *$ & $2.20 *$ & $7.83^{*}$ & $11.43^{*}$ & $0.17 *$ & $0.20 *$ & $0.22 *$ & $0.09 *$ & $1.88^{*}$ & $1.63^{*}$ & $2.63^{*}$ & $1.03 *$ \\
\hline \multicolumn{13}{|c|}{ Nitrogen levels $(\mathbf{N})$} \\
\hline $\mathrm{N}_{1}$ & $31.92 b$ & 32.39 & $142 b$ & $168 \mathrm{a}$ & $3.35 \mathrm{a}$ & $3.40 \mathrm{a}$ & $3.49 \mathrm{a}$ & $3.24 \mathrm{a}$ & $64.65 \mathrm{a}$ & $79.98 \mathrm{a}$ & 18.61 & 18.99 \\
\hline $\mathrm{N}_{2}$ & $32.53 b$ & 34.32 & $161 \mathrm{a}$ & $161 b$ & $3.26 \mathrm{a}$ & $3.42 \mathrm{a}$ & $2.98 \mathrm{~b}$ & $3.39 \mathrm{~b}$ & $39.27 b$ & $41.07 \mathrm{~b}$ & 18.51 & 18.80 \\
\hline $\mathrm{N}_{3}$ & $38.44 a$ & 33.27 & $163 \mathrm{a}$ & $142 \mathrm{c}$ & $2.96 \mathrm{~b}$ & $3.10 \mathrm{~b}$ & $3.16 \mathrm{~b}$ & $3.72 b$ & $22.62 \mathrm{c}$ & $27.38 \mathrm{c}$ & 18.11 & 18.24 \\
\hline LSD $(\mathrm{P}<0.05)$ & $2.32 *$ & NS & $6.78 *$ & $9.90 *$ & $0.15^{*}$ & $0.17 *$ & $0.22 *$ & $0.162 *$ & $1.63 *$ & 1.41 & NS & NS \\
\hline \multicolumn{13}{|l|}{ Genotypes (V) } \\
\hline $\mathrm{V}_{1}$ & $37.96 \mathrm{a}$ & $35.18 \mathrm{a}$ & $163 a$ & $178 \mathrm{a}$ & $3.60 \mathrm{a}$ & $3.76 \mathrm{a}$ & $3.08 \mathrm{~b}$ & $3.03 \mathrm{~b}$ & 42.13 & $49.64 \mathrm{a}$ & $20.20 \mathrm{a}$ & $19.69 \mathrm{a}$ \\
\hline $\mathrm{V}_{2}$ & $31.37 \mathrm{~b}$ & $33.44 \mathrm{a}$ & $168 \mathrm{a}$ & $163 b$ & $2.91 b$ & $2.96 \mathrm{c}$ & $2.99 \mathrm{~b}$ & $3.24 b$ & 42.12 & $51.00 \mathrm{a}$ & $17.54 \mathrm{ab}$ & $17.95 b$ \\
\hline $\mathrm{V}_{3}$ & $33.56 b$ & $31.35 b$ & $134 b$ & $130 \mathrm{c}$ & $3.06 \mathrm{~b}$ & $3.21 \mathrm{~b}$ & $3.57 \mathrm{a}$ & $4.07 \mathrm{a}$ & 42.29 & $47.79 b$ & $17.48 b$ & $18.38 b$ \\
\hline LSD $(\mathrm{P}<0.05)$ & $2.32 *$ & $1.90^{*}$ & $6.78 *$ & $9.90 *$ & $0.15^{*}$ & $0.17 *$ & $0.20 *$ & $0.162 *$ & NS & 1.41 & $2.07 *$ & $0.89 *$ \\
\hline \multicolumn{13}{|l|}{ Interaction $^{\wedge \wedge}$} \\
\hline $\mathrm{I} \times \mathrm{N}$ & NS & $2.61 *$ & 27.10 & $9.80 *$ & $0.30 *$ & $0.35^{*}$ & $0.20 *$ & $0.16^{*}$ & $3.26 *$ & $2.82 *$ & $5.84 *$ & $1.78 *$ \\
\hline $\mathrm{I} \times \mathrm{V}$ & NS & $2.19 *$ & $27.10^{*}$ & $9.80 *$ & $0.30 *$ & $0.35 *$ & $0.20 *$ & $0.16^{*}$ & $3.26 *$ & $2.83 *$ & $5.84 *$ & $1.78 *$ \\
\hline $\mathrm{N} \times \mathrm{V}$ & NS & NS & $23.47 *$ & $7.15^{*}$ & $0.26 *$ & $0.30 *$ & $0.17 *$ & $0.14 *$ & $2.82 *$ & $2.45 *$ & $4.79 *$ & $1.54 *$ \\
\hline $\mathrm{I} \times \mathrm{N} \times \mathrm{V}$ & $8.04 *$ & $6.59 *$ & $6.94 *$ & $4.30 *$ & $0.51 *$ & $0.61 *$ & $0.34 *$ & $0.28 *$ & $5.64 *$ & $4.89 *$ & $11.99 *$ & $3.08 *$ \\
\hline Grand mean & 34.29 & 33.32 & 155.3 & 57.00 & 3.19 & 3.31 & 3.21 & 3.45 & $42.18 *$ & 49.48 & 18.41 & 18.67 \\
\hline $\mathrm{CV}$ & 14.39 & 12.14 & 9.29 & 6.73 & 9.89 & 11.25 & 13.08 & 10.00 & 8.22 & 6.07 & 19.93 & 10.16 \\
\hline
\end{tabular}

*Factor/treatment effect is significant at $\mathrm{P} \leq 0.05, \mathrm{NS}=$ not-significant at $\mathrm{P} \leq 0.05$; Values within a column (factor wise)

followed by the same letter are not statistically different at $\mathrm{P} \leq 0.05 ; \wedge \wedge$ LSD values at $\mathrm{P} \leq 0.05 ; \mathrm{d}=\mathrm{days}$ 
during 2008-09 in $\mathrm{I}_{4} \times \mathrm{N}_{3} \times \mathrm{V}_{3}$ and $\mathrm{I}_{4} \times \mathrm{N}_{3} \times \mathrm{V}_{1}$ treatments, respectively. Similar trend was observed during 2009-10 when lowest LAD was found as 79 and 83 by $\mathrm{I}_{4} \times \mathrm{N}_{3} \times \mathrm{V}_{3}$ and $\mathrm{I}_{4} \times \mathrm{N}_{1} \times \mathrm{V}_{3}$, respectively (as shown in Fig. $1 \mathrm{c}, \mathrm{d}$ ).

\section{a. Number of bolls per plant during 2008-09}

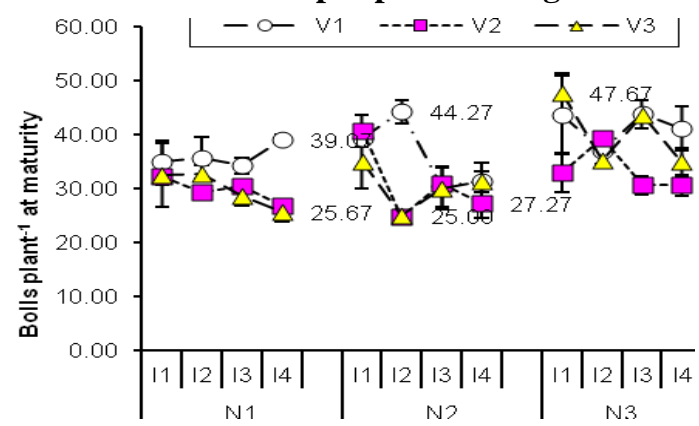

LSD: 8.04, CV: 14.39, Grand mean: 34.29

c. Leaf area duration during 2008-09

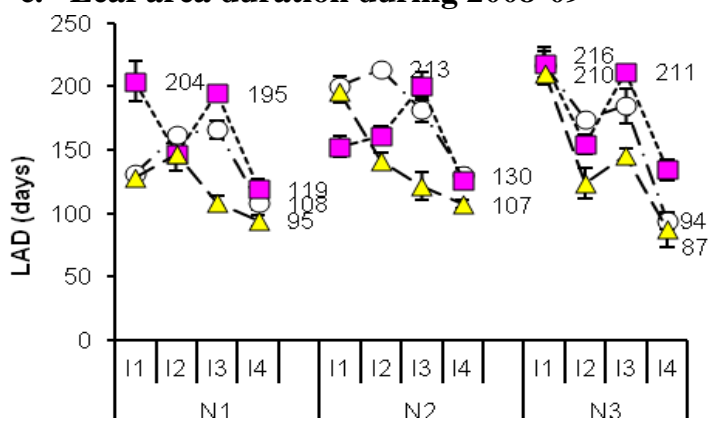

LSD: 6.94, CV: 9.29, Grand mean: 155.3

e. Crop growth rate during 2008-09

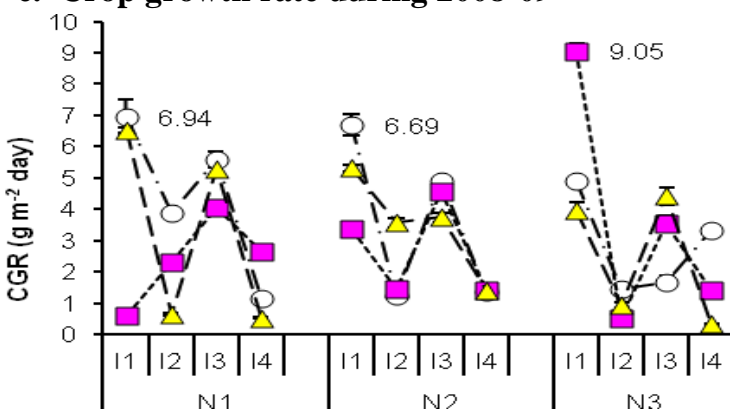

LSD: 0.51, CV: 9.89, Grand mean: 3.19
Crop growth rate: Both the year crop growth rate (CGR) varied significantly by nitrogen and moisture stress treatments as shown in Table 2. During 2008-09, highest CGR of $9.05 \mathrm{~g}$ $\mathrm{m}^{-2} \mathrm{~d}^{-1}$ was recorded (Fig. 1e) in treatment $\mathrm{I}_{1} \times \mathrm{N}_{3} \times \mathrm{V}_{2}$ (no stress $\left.\times 150 \mathrm{~kg} \mathrm{ha}^{-1} \mathrm{~N} \times \mathrm{NIAB}-824\right)$, followed by CGR of 6.94 and

b. Number of bolls per plant during 2009-10

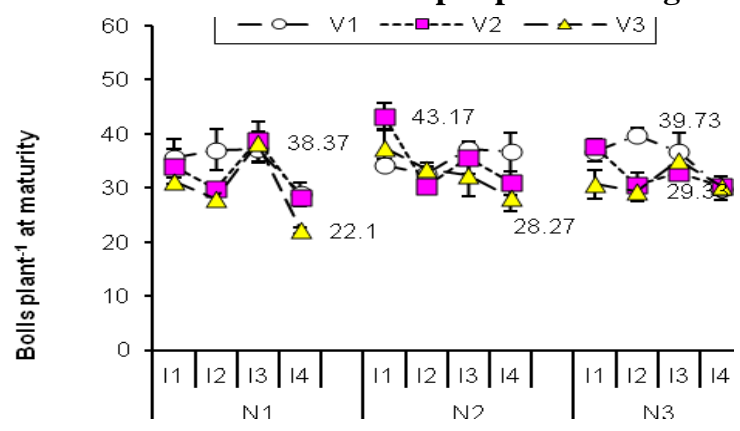

LSD: 6.59, CV: 12.14, Grand mean: 33.32

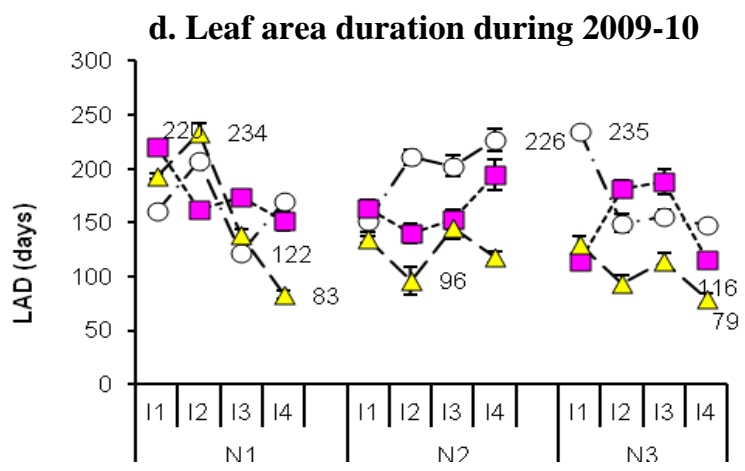

LSD: 4.30, CV: 6.73, Grand mean: 57.00

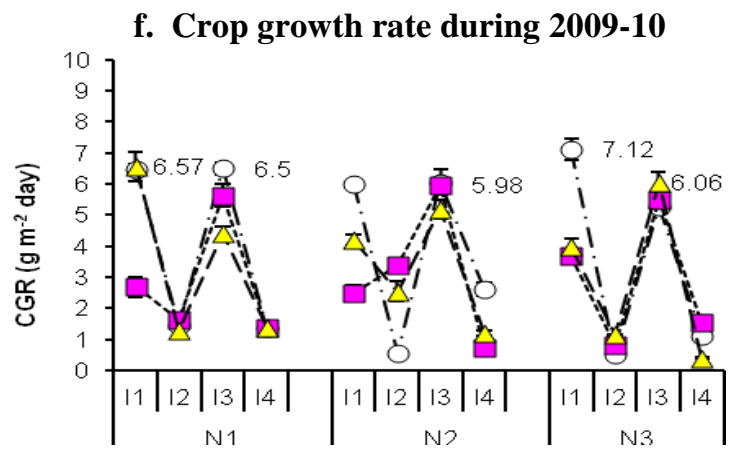

LSD: 0.61, CV: 11.25, Grand mean: 3.31

Figure 1. Interaction effect of moisture stress, nitrogen, and genotypes on; number of bolls per plant (a, b), leaf area duration (c, d), and crop growth rate $(\mathbf{e}, \mathbf{f})$. Factors are; moisture stress (I), nitrogen (N), and genotypes (V). Whereas moisture stress levels are; $I_{1}=$ no stress $=$ water application at $50 \%$ available soil moisture depletion level (eight irrigations); $I_{2}=$ moisture stress at inter-node elongation stage at $80 \%$ ASMDL; $I_{3}=$ moisture stress at vegetative growth stage at $80 \%$ ASMDL; $\mathrm{I}_{4}=$ moisture stress at inter-node elongation and vegetative growth stage at $80 \%$ ASMDL; nitrogen levels are; $\mathrm{N}_{1}=50 \mathrm{~kg} \mathrm{~N} \mathrm{ha}^{-1}, \mathrm{~N}_{2}=100 \mathrm{~kg} \mathrm{~N} \mathrm{ha}^{-1}$, and $\mathrm{N}_{3}=150 \mathrm{~kg} \mathrm{~N} \mathrm{ha}{ }^{-1}$; genotypes are; $\mathrm{V}_{1}=$ NIAB-846, $\mathrm{V}_{2}=$ NIAB-824, $\mathrm{V}_{3}=\mathrm{CIM}-496$. 
$6.69 \mathrm{~g} \mathrm{~m}^{-2} \mathrm{~d}^{-1}$ in treatments $\mathrm{I}_{1} \times \mathrm{N}_{1} \times \mathrm{V}_{1}$ (no stress $\times 50 \mathrm{~kg} \mathrm{ha}^{-1}$ $\mathrm{N} \times \mathrm{NIAB}-846$ ) and $\mathrm{I}_{1} \times \mathrm{N}_{2} \times \mathrm{V}_{1}$ (no stress $\times 100 \mathrm{~kg} \mathrm{ha}^{-1} \mathrm{~N} \times$ NIAB-846), respectively. Lowest CGR of $0.33 \mathrm{~g} \mathrm{~m}^{-2} \mathrm{~d}^{-1}$ and $0.50 \mathrm{~g} \mathrm{~m}^{-2} \mathrm{~d}^{-1}$ in treatments $\mathrm{I}_{4} \times \mathrm{N}_{3} \times \mathrm{V}_{3}$ (moisture stress at internodes elongation and vegetative growth stages $\times 150 \mathrm{~kg}$ ha $^{-1} \mathrm{~N} \times \mathrm{CIM}-496$ ) and $\mathrm{I}_{2} \times \mathrm{N}_{3} \times \mathrm{V}_{2}$ (moisture stress at internodes elongation stage $\times 50 \mathrm{~kg} \mathrm{ha}^{-1} \mathrm{~N} \times \mathrm{NIAB}-824$ ), respectively. In all genotypes, highest crop growth rate was

a. Net assimilation rate during 2008-09

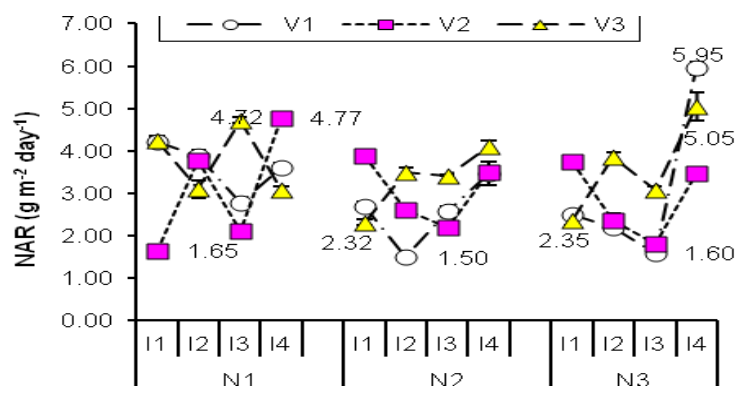

LSD: 0.34, CV: 13.08, Grand mean: 3.21

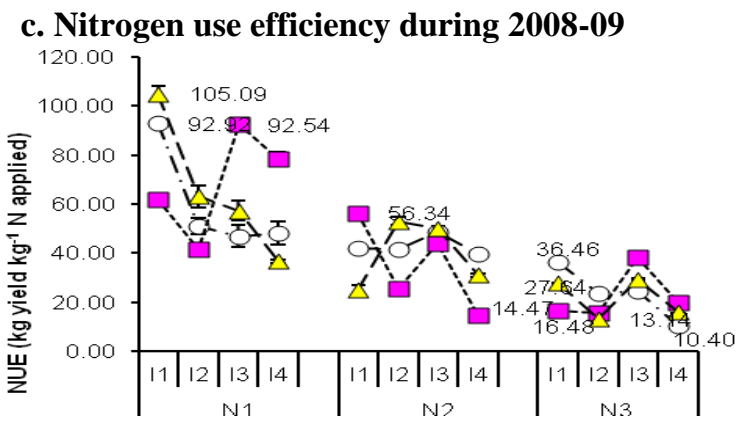

LSD: 5.64, CV: 8.22 , Grand mean: 42.18

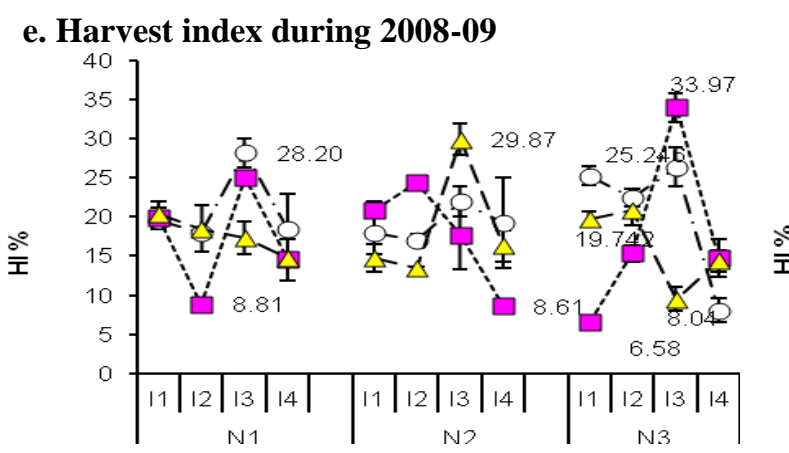

LSD: 1.99, CV: 19.93, Grand mean: 18.41 recorded in treatments of where no stress and moisture stress at vegetative growth stage with $\mathrm{N}$ dose of $50 \mathrm{~kg} \mathrm{ha}^{-1}$ was used expect in few treatments where $150 \mathrm{~kg} \mathrm{~N} \mathrm{ha}^{-1}$ exhibited greater crop growth rate (Fig. 1e,f). Lowest CGR, $0.40 \mathrm{~g} \mathrm{~m}^{-2}$ $\mathrm{d}^{-1}$ and $0.54 \mathrm{~g} \mathrm{~m}^{-2} \mathrm{~d}^{-1}$ was observed in $\mathrm{I}_{4} \times \mathrm{N}_{3} \times \mathrm{V}_{3}$ (moisture stress at internodes elongation and vegetative growth stages $\times$ $150 \mathrm{~kg} \mathrm{~N} \mathrm{ha}^{-1} \times$ CIM-496) and $\mathrm{I}_{2} \times \mathrm{N}_{3} \times \mathrm{V}_{1}(\mathrm{MSI} \times 150 \mathrm{~kg} \mathrm{~N}$ $\mathrm{ha}^{-1} \times$ NIAB-846), respectively.

b. Net assimilation rate during 2009-10

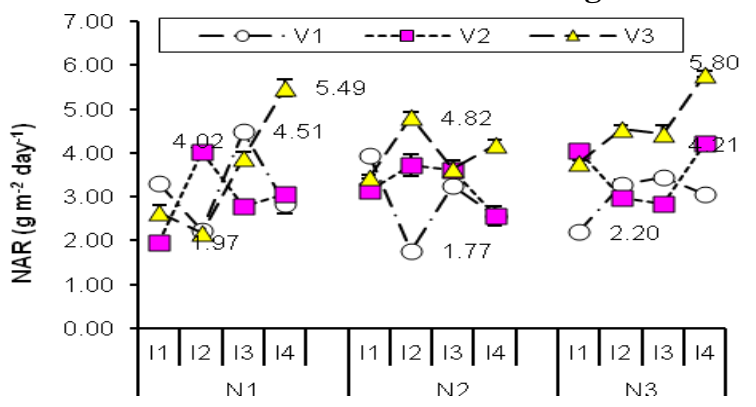

LSD: $0.28, \mathrm{CV}: 10.00$, Grand mean: 3.45

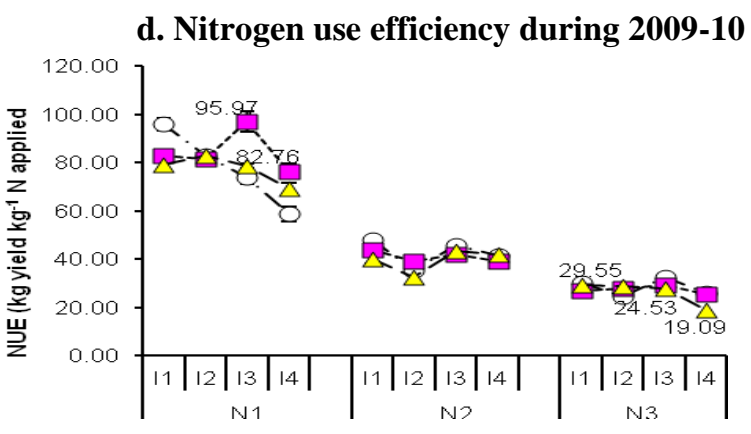

LSD: $4.89, \mathrm{CV}: 6.07$, Grand mean: 49.48

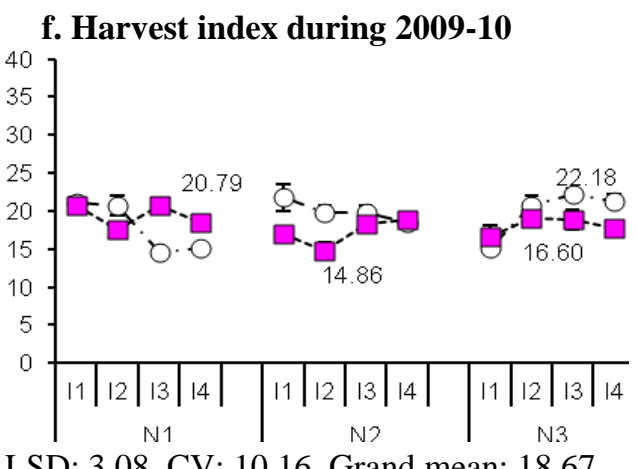

Figure 2. Interaction effect of moisture stress, nitrogen, and genotypes on; net assimilation rate (a, b), nitrogen use efficiency (c, d), and harvest index (e, f) of cotton. Factors are; moisture stress (I), nitrogen (N), and genotypes $(\mathrm{V})$. Whereas moisture stress levels are; $\mathrm{I}_{1}=$ no stress $=$ water application at $50 \%$ available soil moisture depletion level (eight irrigations); $\mathrm{I}_{2}=$ moisture stress at inter-node elongation stage at $80 \%$ ASMDL; $\mathrm{I}_{3}=$ moisture stress at vegetative growth stage at $80 \%$ ASMDL; $\mathrm{I}_{4}=$ moisture stress at inter-node elongation and vegetative growth stage at $80 \%$ ASMDL; nitrogen levels are; $\mathrm{N}_{1}=50 \mathrm{~kg} \mathrm{~N} \mathrm{ha}^{-1}, \mathrm{~N}_{2}=100 \mathrm{~kg} \mathrm{~N}^{-1}$, and $\mathrm{N}_{3}=$ $150 \mathrm{~kg} \mathrm{~N}^{-1}$; genotypes are; $\mathrm{V}_{1}=$ NIAB-846, $\mathrm{V}_{2}=$ NIAB-824, $\mathrm{V}_{3}=\mathrm{CIM}-496$. 
Net assimilation rate: Factors and treatments affected net assimilation rate (NAR) and it has been shown in Table 2 and Figure 2a,b. As shown in Fig. 2a, during 2008-09 highest NAR was recorded by $\mathrm{I}_{4} \times \mathrm{N}_{3} \times \mathrm{V}_{3}$ (moisture stress at internodes elongation and vegetative growth stage $\times 150 \mathrm{~kg} \mathrm{~N}^{-1} \times$ NIAB-846) with 5.95 and 5.05 NAR in $\mathrm{I}_{4} \times \mathrm{N}_{3} \times \mathrm{V}_{3}$ and $\mathrm{I}_{4} \times \mathrm{N}_{1} \times \mathrm{V}_{2}$, treatments, respectively. Lowest NAR of 1.50 and $1.60 \mathrm{~g} \mathrm{~m}^{-2}$ day $^{-1}$ was recorded in treatments $\mathrm{I}_{2} \times \mathrm{N}_{2} \times \mathrm{V}_{1}$ (moisture stress at internodes elongation stage $\times 100 \mathrm{~kg} \mathrm{~N}^{-}$ ${ }^{1} \times \mathrm{NIAB}-846$ ), and $\mathrm{I}_{3} \times \mathrm{N}_{3} \times \mathrm{V}_{1}$ (moisture stress at vegetative growth stage $\times 150 \mathrm{~kg} \mathrm{~N} \mathrm{ha}^{-1} \times$ NIAB-846), respectively. Fig. $2 \mathrm{~b}$ shows treatments effect on NAR during 2009-10, highest NAR 5.80, 5.49, and $4.82 \mathrm{~g} \mathrm{~m}^{-2}$ day $^{-1}$ were recorded by $\mathrm{I}_{4} \times \mathrm{N}_{3} \times \mathrm{V}_{3}$ (moisture stress at internodes elongation and vegetative growth stages $\times 150 \mathrm{~kg} \mathrm{ha}^{-1} \mathrm{~N} \times$ CIM-496), $\mathrm{I}_{4} \times \mathrm{N}_{1} \times \mathrm{V}_{3}\left(\mathrm{MSI}+\mathrm{MSV} \times 50 \mathrm{~kg} \mathrm{ha}^{-1} \mathrm{~N} \times \mathrm{CIM}-496\right)$, and $\mathrm{I}_{2}$ $\times \mathrm{N}_{2} \times \mathrm{V}_{3}\left(\mathrm{MSI} \times 100 \mathrm{~kg} \mathrm{ha}^{-1} \mathrm{~N} \times\right.$ CIM-496) treatments, respectively. Lowest, $1.77,1.97$, and $2.20 \mathrm{~g} \mathrm{~m}^{-2}$ day $^{-1} \mathrm{NAR}$ were observed in $\mathrm{I}_{2} \times \mathrm{N}_{2} \times \mathrm{V}_{1}$ (moisture stress at internodes elongation stage $\left.\times 100 \mathrm{~kg} \mathrm{ha}^{-1} \mathrm{~N} \times \mathrm{NIAB}-846\right), \mathrm{I}_{1} \times \mathrm{N}_{1} \times \mathrm{V}_{2}$ (no stress $\times 50 \mathrm{~kg} \mathrm{ha}^{-1} \mathrm{~N} \times \mathrm{NIAB}-824$ ), and $\mathrm{I}_{1} \times \mathrm{N}_{3} \times \mathrm{V}_{1}$ (no stress $\left.\times 150 \mathrm{~kg} \mathrm{ha}^{-1} \mathrm{~N} \times \mathrm{NIAB}-846\right)$, respectively.

Nitrogen use efficiency: Nitrogen use efficiency (NUE) in cotton genotypes as influenced by factors and treatments are shown in Table 2. Main effects results show higher NUE by no stress, and moisture stress at vegetative growth stage. Nitrogen dose $150 \mathrm{~kg} \mathrm{ha}^{-1}$ showed higher NUE. As shown in Figure 2c, interaction of moisture stress, $\mathrm{N}$ levels, and genotypes $(\mathrm{I} \times \mathrm{N} \times \mathrm{V})$ significantly affected NUE during 2008 09, with highest NUE of 105.09, 92.92, and $92.54 \mathrm{~kg}$ seed cotton yield (SCY) $\mathrm{kg}^{-1} \mathrm{~N}$ by $\mathrm{I}_{1} \times \mathrm{N}_{1} \times \mathrm{V}_{3}$ (no stress $\times 50 \mathrm{~kg} \mathrm{ha}^{-}$ $\left.{ }^{1} \mathrm{~N} \times \mathrm{CIM}-496\right), \mathrm{I}_{1} \times \mathrm{N}_{1} \times \mathrm{V}_{1}$ (no stress $\times 50 \mathrm{~kg} \mathrm{ha}^{-1} \mathrm{~N} \times \mathrm{NIAB}-$ 846), and $I_{3} \times N_{1} \times V_{2}$ (moisture stress at vegetative growth stage $\times 50 \mathrm{~kg} \mathrm{ha}^{-1} \mathrm{~N} \times$ NIAB-824) treatments, respectively. Lowest NUE, 10.40 and $13.14 \mathrm{~kg} \mathrm{SCY} \mathrm{kg}^{-1} \mathrm{~N}$ was recorded in $\mathrm{I}_{4} \times \mathrm{N}_{3} \times \mathrm{V}_{1}$ (moisture stress at internodes elongation and vegetative growth stage $\times 150 \mathrm{~kg} \mathrm{ha}^{-1} \mathrm{~N} \times \mathrm{NIAB}-846$ ), and $\mathrm{I}_{2} \times \mathrm{N}_{3} \times \mathrm{V}_{3}$ (moisture stress at internodes elongation stage $\times$ $150 \mathrm{~kg} \mathrm{ha}^{-1} \mathrm{~N} \times$ CIM-496) treatments, respectively. As shown in Fig. 2d, during 2009-10 highest NUE 97.06 and $95.97 \mathrm{~kg}$ SCY $\mathrm{kg}^{-1} \mathrm{~N}$ were recorded by $\mathrm{I}_{3} \times \mathrm{N}_{1} \times \mathrm{V}_{2}$ (moisture stress at vegetative growth stage $\left.\times 50 \mathrm{~kg} \mathrm{ha}^{-1} \mathrm{~N} \times \mathrm{NIAB}-824\right)$ and $\mathrm{I}_{1} \times \mathrm{N}_{1} \times \mathrm{V}_{1} \quad$ (no stress $\times 50 \mathrm{~kg} \mathrm{ha}^{-1} \mathrm{~N} \times$ NIAB-846), respectively.

Harvest index: Results showed (Table 2) that moisture stress, nitrogen, and genotypic interaction significantly affected harvest index (HI). During 2008-09 and 2009-10, highest HI of $23.32 \%$ and $19.68 \%$ was recorded in the moisture stress treatment at vegetative stage $\left(\mathrm{I}_{3}\right)$. NIAB-846 showed highest HI of 20.20\% during 2008-09 and during 2009-10 highest HI of $17.95 \%$ was recorded NIAB-824. Minimum HI of 14.35 and $17.90 \%$ was observed in $\mathrm{I}_{4}$ (moisture stress at internodes elongation and vegetative growth stage). Treatments $(\mathrm{I} \times \mathrm{N} \times \mathrm{V})$ results shown in Fig. 2e,f indicate a significant effect on $\mathrm{HI}$ by interaction effect of factors. Highest HI of $33.97 \%$ during 2008-09 (Fig. 2e) was recorded in $\mathrm{I}_{3} \times \mathrm{N}_{3} \times \mathrm{V}_{2}$ (moisture stress at vegetative growth stage $\times 150 \mathrm{~kg} \mathrm{~N}^{-1} \times$ NIAB-824) followed by $28.87 \% \mathrm{HI}$ in $\mathrm{I}_{3} \times \mathrm{N}_{2} \times \mathrm{V}_{3}$ (moisture stress at vegetative growth stage $\times 100 \mathrm{~kg} \mathrm{~N} \mathrm{ha}^{-1} \times$ CIM-496). During 2009-10 (Fig. 2f), treatment $\mathrm{I}_{1} \times \mathrm{N}_{2} \times \mathrm{V}_{3}$ (no stress $\times 100 \mathrm{~kg} \mathrm{~N}$ ha $^{-1} \times$ CIM-496) resulted with $25.07 \% \mathrm{HI}$ followed by $22.18 \% \mathrm{HI}$ in $\mathrm{I}_{3} \times \mathrm{N}_{3} \times \mathrm{V}_{1}$ (moisture stress at vegetative growth stage $\times 150 \mathrm{~kg} \mathrm{~N}^{-1} \times$ NIAB-846) treatment. Minimum HI (during 2008-09) of $6.58 \%$ and $8.05 \%$ was observed by treatments $\mathrm{I}_{1} \times \mathrm{N}_{3} \times \mathrm{V}_{2}$ (no stress $\left.\times 150 \mathrm{~kg} \mathrm{~N} \mathrm{ha}^{-1} \times \mathrm{NIAB}-824\right)$ and $\mathrm{I}_{4} \times \mathrm{N}_{3} \times \mathrm{V}_{1}$ (moisture stress at internodes elongation and vegetative growth stages $\times 150 \mathrm{~kg} \mathrm{~N}^{-1} \times$ NIAB-846), respectively. During 2009-10, lowest HI of $14.86 \%, 15.17 \%$, and $15.59 \%$ was recorded in treatments $\mathrm{I}_{2} \times \mathrm{N}_{2} \times \mathrm{V}_{2}$ (moisture stress at internodes elongation stage $\times 100 \mathrm{~kg} \mathrm{~N}^{-1} \times \mathrm{NIAB}-$ $824), \mathrm{I}_{1} \times \mathrm{N}_{3} \times \mathrm{V}_{1}$ (no stress $\left.\times 150 \mathrm{~kg} \mathrm{~N} \mathrm{ha}^{-1} \times \mathrm{NIAB}-846\right)$, and $\mathrm{I}_{4} \times \mathrm{N}_{3} \times \mathrm{V}_{3}$ (moisture stress at internodes elongation and vegetative growth stages $\times 150 \mathrm{~kg} \mathrm{~N} \mathrm{ha}^{-1} \times$ CIM-496), respectively.

\section{DISCUSSION}

Water scarcity due to global environmental changes is drastically affecting the seed cotton production. This scenario has opened up a challenge for agronomists to develop new technologies. Moisture stress along with nitrogen management at certain growth stages of cotton is found a promising approach for saving of irrigation water and urea fertilizer. Plant growth, nitrogen use efficiency and harvest index of cotton is improved by imposing moisture stress at vegetative growth stage coupled with lower N. Prior to this study, Meredith et al. (1997) noted highest yield in cotton genotypes by lower $\mathrm{N}$ application as compared to high $\mathrm{N}$ dose.

Field experiments were conducted (during 2008-2010) to investigate the impact of interaction of moisture stress, nitrogen, and genotypes on growth, nitrogen use efficiency, and harvest index of cotton. Higher number of bolls per plant was observed in treatments of; no moisture stress, and moisture stress at vegetative growth stage. Number of bolls per plant were significantly reduced in cotton genotypes by $\mathrm{I}_{4}$ and $\mathrm{I}_{2}$. Results showed that NIAB-846 got sustainable fruiting by different $\mathrm{N}$ doses used exhibiting more stability towards multiple stresses where as other two genotypes showed inconsistent trend in fruit bearing by application of different $\mathrm{N}$ doses. Mixed response of cotton genotypes towards fruit bearing by $\mathrm{N}$ application indicates that deteriorating and/or adaptive changes in cotton plants under multiple interaction effects in the field, is genotypic-specific which depends upon morphological response of specific genotypes. The results in the present study show that highest number of bolls per plant was recorded by lower application of $\mathrm{N}\left(100 \mathrm{~kg} \mathrm{~N} \mathrm{ha}^{-1}\right)$ in NIAB-824 as compared to higher $\mathrm{N}$ (150 $\left.\mathrm{kg} \mathrm{ha}^{-1}\right)$ application 
during 2009-10 as shown in Fig. 1a. Highest number of bolls per plant (Fig. 1b), was recorded in CIM-496 by application of $150 \mathrm{~kg} \mathrm{ha}^{-1} \mathrm{~N}$ in no stress treatment. In previous studies (Milroy and Bange, 2004), N deficiency had been reported as a limiting factor for vegetative growth and fruiting. In some other studies it had been reported that higher level of $\mathrm{N}$ application cause more vegetative growth while shifting the balance between reproductive and vegetative growth phases and causing less fruiting (Howard et al., 2001). Sensitivity of cotton to $\mathrm{N}$ application is well established; excessive as well as lower $\mathrm{N}$ dose both may cause drastic effects causing higher vegetative growth, less fruiting, and ultimately lower seed cotton yield. Field experiments resembling the present investigation will help to sort-out best genotypic-specific $\mathrm{N}$ and moisture stress combinations for achievement of better fruiting and higher seed cotton yield.

The results of leaf area duration (LAD) are presented in Figure 1c,d. Lowest LAD was observed in high moisture stress treatments (i.e. moisture stress at internodes elongation and at vegetative growth stages). In all genotypes LAD was not affected by $\mathrm{N}$ levels in $\mathrm{I}_{4}$ stress level. However, LAD was increased with higher $\mathrm{N}$ application in no stress treatment. Improvement in LAD was also note in treatments where moisture stress was imposed at vegetative growth stage. Higher seed cotton yield was observed in the treatments where more LAD was achieved due to increase in fruiting and crop growth rate. Wolfe et al. (1983) also studied nitrogen and moisture stress impact on LAD of maize where they found an inhibition in LAD, crop biomass, and ultimately grain yield by deficit irrigation and $\mathrm{N}$ application. In present study we have observed that deficit irrigation at vegetative growth stage of cotton didn't markedly inhibited LAD and seed cotton yield as compared to well water (no stress) treatment, even by application of lower $\mathrm{N}$ dose. This technique may be helpful in saving of irrigation water and $\mathrm{N}$ fertilizer.

Highest crop growth rate was achieved by well water (no stress) treatments as shown in Figure 1e,f. The cotton genotypes grown under no stress (well water) treatments showed inconsistent response towards different $\mathrm{N}$ doses. Higher CGR trend was observed in NIAB-846 by lower $\mathrm{N}$ dose $\left(50 \mathrm{~kg} \mathrm{ha}^{-1}\right)$, whereas higher CGR in other genotypes, CIM-496 and NIAB-824, was noted with higher $\mathrm{N}$ doses of $100,150 \mathrm{~kg} \mathrm{~N} \mathrm{ha}^{-1}$, respectively. Lowest CGR was recorded in high stress treatments (moisture stress at internodes elongation and vegetative growth stages). In earlier studies, Monteith (1976) reported that radiation use efficiency and CGR in cotton was reduced under drought conditions. In another study made by McMichael and Hesketh (1982), it was observed that induced drought in cotton (Gossypium hirsutum L.) caused a reduction in photosynthesis rate, leaf expansion, vegetative nodes, and CGR. However, the information about the changes in CGR by multiple interaction effect of moisture stress at certain growth stages of cotton, $\mathrm{N}$ doses, and genotypes; was missing.
Net assimilation rate was significantly affected by moisture stress treatments as shown in Figure 2a,b. Highest NAR was observed in high moisture stress treatments (moisture stress at internodes and vegetative growth stages) along with higher $\mathrm{N}$ doses $\left(100,150 \mathrm{~kg} \mathrm{ha}^{-1}\right)$. However, in no stress (well water) treatment highest NAR was noted when lower N dose $(50 \mathrm{~kg}$ $\mathrm{ha}^{-1}$ ) was applied. Kimball and Mauney (1993) studied the effect of moisture stress, $\mathrm{N}$, and $\mathrm{CO}_{2}$ levels on cotton and they observed 60-63\% increase in cotton yield in moisture stress treatments as compared to control (no stress) treatment. They also found that under well managed moisture stress, $\mathrm{CO}_{2}$ response was higher as compared to normal irrigation levels. In present study, higher NAR values were recorded in moisture stress treatments without any change in the $\mathrm{CO}_{2}$ level and under no stress (normal irrigation); lower $\mathrm{N}$ dose resulted in higher NAR.

In cotton genotypes highest NUE was attained by $\mathrm{N}$ interaction with no stress (well water) and moisture stress at vegetative growth stage. In the treatments of vegetative growth stage stress, even lower $\mathrm{N}$ dose $\left(50 \mathrm{~kg} \mathrm{ha}^{-1}\right)$ gave higher NUE as shown in Figure 2c,d. Thind et al. (2008) investigated irrigation and $\mathrm{N}$ effect on cotton and found significant effect of treatments on NUE of cotton but he didn't evaluated the comparative NUE in different genotypes of cotton. While comparing highest NUE in three genotypes over control treatments $\left(\mathrm{I}_{1} \times \mathrm{N}_{3} \times \mathrm{V}_{1}, \mathrm{I}_{1} \times \mathrm{N}_{3} \times \mathrm{V}_{2}, \mathrm{I}_{1} \times \mathrm{N}_{3} \times \mathrm{V}_{3}\right)$, during 2008-09 (Fig. 2c), 60.76\% higher NUE was noted in NIAB-846 in treatment $\mathrm{I}_{1} \times \mathrm{N}_{1} \times \mathrm{V}_{1}$ (no stress $\times 50 \mathrm{~kg} \mathrm{~N} \mathrm{ha}^{-1} \times$ NIAB-846, $82.19 \%$ higher NUE in NIAB-824 in treatment $\mathrm{I}_{3} \times \mathrm{N}_{1} \times \mathrm{V}_{2}$ (moisture stress at vegetative growth stage $\times 50 \mathrm{~kg}$ $\left.\mathrm{N} \mathrm{ha}{ }^{-1} \times \mathrm{NIAB}-824\right)$, and $73.86 \%$ higher NUE in CIM-496 in treatment $\mathrm{I}_{1} \times \mathrm{N}_{1} \times \mathrm{V}_{3}$ (no stress $\times 50 \mathrm{~kg} \mathrm{~N}^{-1} \times \mathrm{CIM}-496$ ). Similar trend was observed during 2009-10 (Fig. 2d) where $69.20 \%$ higher NUE was noted in NIAB-846 in $\mathrm{I}_{1} \times \mathrm{N}_{1} \times \mathrm{V}_{1}$ treatment, $72.06 \%$ higher NUE in NIAB-824 in $\mathrm{I}_{3} \times \mathrm{N}_{1} \times \mathrm{V}_{2}$ treatment, and $64.29 \%$ higher NUE in CIM-496 in $\mathrm{I}_{2} \times \mathrm{N}_{1} \times \mathrm{V}_{3}$ treatment. In earlier experiments Meredith et al. (1997) also observed effect of $\mathrm{N} \times$ genotypic interaction on NUE of cotton cultivars and they found lower $\mathrm{N}$ dose better as compared to higher $\mathrm{N}$ application. Higher $\mathrm{N}$ application is yield limiting factor in cotton due to shifting of plant balance towards more vegetative growth that delays maturity and lowers seed cotton yield (Howard et al., 2001). In present study we also observed highest NUE in cotton genotypes by lower $\mathrm{N}$ dose $\left(50 \mathrm{~kg} \mathrm{~N} \mathrm{ha}^{-1}\right)$ as compared to higher level of $\mathrm{N}$ $\left(150 \mathrm{~kg} \mathrm{~N} \mathrm{ha}^{-1}\right)$. These results are in line with the previous studies made by Nicholos et al. (2004) who elucidated that excessive use of $\mathrm{N}$ in cotton may be a limiting factor for the growth, yield and radiation use efficiency.

Treatments significantly affected harvest index (HI) of cotton genotypes as shown in Figure 2e,f. During 2008-09 (Fig. 2e), highest HI was noted in treatments where moisture stress was imposed at vegetative growth stage. Highest HI of $33.97 \%$, $29.87 \%$, and $28.20 \%$ was observed in treatments $\mathrm{I}_{3} \times \mathrm{N}_{3} \times \mathrm{V}_{2}$ 
(moisture stress at vegetative growth stage $\times 150 \mathrm{~kg} \mathrm{~N}^{-1} \times$ NIAB-824), $\mathrm{I}_{3} \times \mathrm{N}_{2} \times \mathrm{V}_{3}$ (moisture stress at vegetative growth stage $\left.\times 100 \mathrm{~kg} \mathrm{~N}^{-1} \times \mathrm{CIM}-496\right)$, and $\mathrm{I}_{3} \times \mathrm{N}_{1} \times \mathrm{V}_{1}$ (moisture stress at vegetative growth stage $\left.\times 50 \mathrm{~kg} \mathrm{~N} \mathrm{ha}^{-1} \times \mathrm{NIAB}-846\right)$, respectively. In comparison to control $10.46 \%$ higher HI was noted in $\mathrm{I}_{3} \times \mathrm{N}_{1} \times \mathrm{V}_{1}$ (moisture stress at vegetative growth stage $\times 50 \mathrm{~kg} \mathrm{~N} \mathrm{ha}^{-1} \times \mathrm{NIAB}-846$ ), $80.62 \%$ in $\mathrm{I}_{3} \times \mathrm{N}_{3} \times \mathrm{V}_{2}$ (moisture stress at vegetative growth stage $\left.\times 50 \mathrm{~kg} \mathrm{~N} \mathrm{ha}^{-1} \times \mathrm{NIAB}-846\right)$, higher HI in NIAB-824, and $33.91 \%$ higher HI in CIM-496 was noted. During 2009-10, the increase in HI values over control treatments were, $31.60 \%$ in NIAB-846 (in treatment $\mathrm{I}_{3} \times \mathrm{N}_{3} \times \mathrm{V}_{1}$ ), 20.98\% in NIAB-824 (in treatment $\mathrm{I}_{1} \times \mathrm{N}_{1} \times \mathrm{V}_{2}$ ), and $20.10 \%$ higher in CIM-496 (in treatment $\mathrm{I}_{1} \times \mathrm{N}_{2} \times \mathrm{V}_{3}$ ). In other stress treatments $\left(\mathrm{I}_{2}\right.$ : moisture stress at internodes elongation stage, and $\mathrm{I}_{4}$ : moisture stress at two stagesinternodes elongation and vegetative growth stages), lower HI values were observed as compared to stress at vegetative growth stage $\left(\mathrm{I}_{3}\right)$. In previous studies Steduto et al. (2009) reported that timing of moisture stress leads towards biomass partition and $\mathrm{HI}$ and it may increase or decrease HI depending upon crop growth stage but the information about the extent of change in HI by moisture stress and the identification of specific growth stages of genotypes at which moisture stress is useful, was missing in previous studies. In this study we have identified specific growth stages of cotton as well as genotypic-specific $\mathrm{N}$ dose for getting better crop growth rate, improvement in nitrogen use efficiency, and higher harvest index; leading towards cotton cultivation economically under limited irrigation application.

Conclusions: In cotton, moisture stress at vegetative growth stage coupled with genotypic-specific $\mathrm{N}$ management may be used for saving of irrigation water and urea fertilizer without compromising the harvest index and nitrogen use efficiency as compared to farmers' practice.

Acknowledgements: The work reported is a part of $\mathrm{PhD}$ studies. The authors acknowledge the support of Nuclear Institute for Agriculture and Biology (NIAB), Pakistan Atomic Energy Commission and Department of Agronomy, $\mathrm{UAF}$ for providing facilities for $\mathrm{PhD}$ research work. We are also grateful to the anonymous reviewers for their very useful suggestions to improve this manuscript.

\section{REFERENCES}

Buttar, G.S., M.S. Aujla, H.S. Thind, C.J. Singh and K.S. Saini. 2007. Effect of timing of first and last irrigation on the yield and water use efficiency of cotton. Agric. Water Manage. 89: 236-242.

Clay, D.E., S.A. Clay, Z. Liu and C. Reese. 2001. Spatial variability of $\mathrm{C}-13$ isotopic discrimination in corn (Zea mays). Comm. Soil Sci. Plant Anal. 32.
Dagdelen, N., H. Basal, E. Yilmaz, T. Gurbuz and S. Akcay. 2009. Different drip irrigation regimes affect cotton yield, water use efficiency and fiber quality in western Turkey. Agric. Water Manage. 96: 111-120.

Devkota, M., C. Matius, J.P.A. Lamers, K.D. Sayre, K.P. Devkota, K.P. Gupta, O. Egamberdiev and P.L.G. Vlek. 2013. Combining permanent beds and residue retention with nitrogen fertilization improves crop yields and water productivity in irrigated arid lands under cotton, wheat and maize. Field Crop Res. 149:105-114.

Fereres, E. and M.A. Soriano. 2007. Deficit irrigation for reducing agricultural water use. J. Exp. Bot. 58: 147-158.

French, B.K. and B.J. Legg. 1979. Rothamsted irrigation. 1994. J. Agric. Sci. Camb. 92: 15-37.

Gul, S. N.U. Khan, S. Batool, M.J. Baloch, M. Munir, M. Sajid, A A. Khakwani, S.H. Ghaloo, Z.A. Soomro and S.F. Kazmi. 2014, Genotype by environment interaction and association of morphoyield variables in upland cotton. J. Anim. Plant Sci. 24: 262-271.

Hatfield, J.L., T.J. Saue and J.H. Prueger. 2001. Managing soils to achieve greater water use efficiency: A review. Agron. J. 93: 271-280.

Howard, D.D., C.O. Gwathmey, M.E. Essington, R.K. Roberts and M.D. Mullen. 2001. Nitrogen fertilization of no-till cotton on loess-derived soils. Agron. J. 93:157163.

Hunt. 1978. Plant Growth Analysis. The Institute Biological Studies. Edward Arnold (Pub.) Ltd. 96: 8-38.

Islam, M.K., R. Akmal, I.M. Mominul and S. Dilurba. 2013. Study of stability and adaptability of new cotton (Gossypium hirsutum L.) at different cotton growing regions of Bangladesh. Bangladesh J. Prog. Sci. Tech. 11:191-194.

Kimball, B.A. and J.R. Mauney. 1993. Response of cotton to varying $\mathrm{CO}_{2}$, irrigation, and nitrogen: yield and growth. Agron. J. 85: 706-712.

McMichael, B.L. and J.D. Hesketh. 1982. Field investigations of the response of cotton to water deficits. Field Crops Res. 5: 319-333.

Meredith, W.R., J.J. Heitholt, W.T. Pettigrow and S.T. Jr, Rayburn. 1997. Comparison of obsolete and modern cotton cultivars at two nitrogen levels. Crop Sci. 37: 1453-1457.

Milroy, S.P. and M.P. Bange. 2004. Nitrogen and light responses of cotton photosynthesis and implications for crop growth. Australian Cotton Cooperative Res. Centre, Narrabri, NSW 2390, Australia. Aust. J. Crop Sci. 43: 904-913.

Monteith, J.L. 1976. Spectral distribution of light in leaves and foliage. In: $\mathrm{H}$. Smith (ed.), Light and Plant Development, pp.447-460. Butterworths, London.

Nicholos, S.P., C.E. Snipes and M.A. Jones. 2004. Cotton growth, lint yield, and fibre quality as affected by row spacing and cultivar. J. Cotton Sci. 8: 1-12. 
Penman, H.I. 1970. Woburn irrigation. 1960-8, IV. Design and interpretation. J. Agric. Sci. 75: 69-73.

Singh, Y., S. Rao and P.L. Regar. 2010. Deficit irrigation and nitrogen effects on seed cotton yield, water productivity and yield response factor in shallow soils of semi-arid environment. Agric. Water Manage. 97: 965-970.

Steel, R.G.D. and J.H. Torrie. 1984. Principles and Procedures of Statistics - A Biometrical Approach. McGraw Hill Book Co. Inc New York. pp.172-177.

Tang, H., S.B. Rompani, J.B. Atkins, Y. Zhou, T. Osterwalder and W. Zhong. 2005. Numb proteins specify asymmetric cell fates via an endocytosis- and proteasomeindependent pathway. Mol. Cell Biol. 25: 2899-2909.
Tennakoon, S.B. and S.P. Milroy. 2003. Crop water use and water use efficiency on irrigated cotton farms in Australia. Agric. Water Manage. 61: 179-94.

Thind, H.S., M.S. Aujla and G.S. Buttar. 2008. Response of cotton to various levels of nitrogen and water applied to normal and paired sown cotton under drip irrigation in relation to check-basin. Agric. Water Manage. 95: 25-34.

Wolfe, D.W., E. Fereresy and R.E. Voss. 1983. Growth and yield response of two potato cultivars to various levels of applied water. J. Irrig. Sci 3: 211-222.

Steduto, P., T.C. Hsiao, D. RAES and E. Fereres. 2009. AquaCrop, The FAO crop model to simulate yield response to water: I. Concepts and underlying principles. Agron. J. 101: 426-437. 